\title{
OTHOTOMICS
}

Revista de economía, empresa y sociedad

Dossier «Prevención de riesgos laborales: tendencias en tiempo de crisis»

SEGURIDAD Y SALUD

\section{Impacto de la crisis económica en las condiciones de trabajo y la salud laboral}

\section{Jaume de Montserrat i Nonó; Fmilia Molinero Ruiz; Andrés Ros Pueyo; Julià Nájera Ghico; Jiri Turdy i Moix}

Instituto de Seguridad y Salud Laboral. Departamento de Empresa y Empleo de la Generalitat de Cataluña

RESUMEN El objetivo de este artículo es analizar los efectos que la crisis económica que sufre Cataluña desde el año 2008 ha provocado en las condiciones de empleo y de trabajo, con especial énfasis en los aspectos relacionados con la seguridad y la salud en el trabajo. El impacto de la crisis, aparte del que ha tenido en las personas que han perdido su trabajo, es ya perceptible en un deterioro de las condiciones de trabajo en aspectos como la jornada o la exposición a riesgos ergonómicos y psicosociales.

A pesar de que este deterioro no se ha traducido todavía en un empeoramiento demasiado significativo en los registros de accidentes de trabajo y enfermedades laborales, esto no nos puede conducir al error de pensar que en prevención la crisis no ha causado daños. Por un lado, hay que tomar con prudencia los datos de los registros públicos de siniestralidad laboral, que no permiten por ahora detectar todos los daños a la salud derivados del trabajo, y, por otro, hay que tener en cuenta que el deterioro en las condiciones de trabajo no se traduce de forma inmediata en accidentes de trabajo o enfermedades laborales. Precisamente por ello es necesario tener en cuenta que, ahora que parece que la situación económica comienza a mejorar, es urgente recuperar el terreno perdido en materia de prevención de riesgos laborales.

PALABRAS CLAVE crisis; prevención; seguridad; salud; condiciones; trabajo; riesgo

\section{Impact of the economic crisis on working conditions and occupational health}

ABSTRACT The objective of this article is to analyze the effects that the economic crisis Catalonia suffers since year 2008 has had on employment and working conditions, with special emphasis on issues related to safety and health at work. The impact of the crisis, besides the effect it has had on people who have lost their jobs, is already perceivable in a worsening of working conditions in areas such as working hours or exposure to ergonomic and psychosocial risks. 
Although this deterioration has not yet translated into a significant increase in the register of accidents and work-related illnesses, this cannot lead us to the mistake of thinking that the crisis has not caused damage in the field of prevention. On the one hand, it is necessary to take caution about data from public registers of occupational accidents, which currently do not allow us to detect workrelated illnesses and, on the other hand, it is necessary to take into account that the deterioration in the working conditions does not immediately translate into occupational accidents or diseases. For this reason, it is necessary, now that it seems the economic situation starts to improve, to regain lost ground in terms of risk prevention.

KEYWORDS crisis; occupational health prevention; safety; working conditions; work; risk

\section{Introducción}

La quiebra del banco de inversiones Lehman Brothers, el 15 de septiembre de 2008, se ha erigido a nivel mundial como la fecha que, simbólicamente, marca el inicio de la crisis financiera global que aún padecemos. En nuestro país, sin embargo, hubo otro hecho simbólico del inicio de esta crisis: el expediente de regulación de empleo en la planta de Nissan de la Zona Franca de Barcelona, que se anunció en octubre del mismo año y que afectaba a más de 1.500 trabajadores. Solo este caso ya nos sirve para mostrar una de las peculiaridades de la crisis en Cataluña y en España con respecto a otros lugares: esta crisis ha sido financiera, sí, pero ha golpeado, y de manera muy dura, al mundo del trabajo.

Ahora que la crisis económica va camino de los siete años, es necesario hacer balance de cuál ha sido el efecto de este largo periodo de dificultades económicas para las empresas y las personas en relación con el mantenimiento del puesto de trabajo y de las condiciones de trabajo, así como de la seguridad y la salud de los trabajadores.

En primer lugar, hay que aclarar que nos referiremos al impacto de la crisis en las condiciones de trabajo, es decir, en todos aquellos aspectos (materiales, pero también organizativos) que inciden en la seguridad, la salud y el bienestar de los trabajadores. Los registros públicos en materia de accidentes de trabajo y enfermedades profesionales no son suficientes para hacer este análisis, dado que solo recogen (y lo hacen parcialmente) los efectos de las condiciones de trabajo, pero no los cambios que estas sufren, que además pueden tardar mucho tiempo en producir consecuencias. El "periodo de latencia» entre los cambios en las condiciones de trabajo y su manifestación en forma de daños a la salud de los trabajadores, tal como ocurre en muchas enfermedades laborales, puede ser de muchos años.

\section{Los más perjudicados}

Antes de considerar los efectos que la crisis ha tenido en el seno de las empresas, es necesario recordar que los efectos más perjudiciales de la crisis en términos de salud y bienestar no los han sufrido los empresarios, ni los autónomos, ni los trabajadores que se mantienen ocupados, sino precisamente aquellos trabajadores que han perdido su trabajo, los empresarios que han perdido su empresa o los autónomos que han tenido que cesar en su actividad (Regulies, 2008).

Los efectos de la crisis en estos colectivos, en particular para las personas que han encontrado y encuentran más dificultades para volver a la vida activa, han sido en muchos casos devastadores. En los últimos años se han publicado varios estudios que relacionan directamente la crisis económica y la angustia que generan el paro y la pérdida de ingresos. También se ha producido en nuestra sociedad un importante aumento de la incidencia de depresiones y otros trastornos mentales, o del consumo de antidepresivos y ansiolíticos. 
Aparte de eso, sin embargo, hay estudios que relacionan procesos de reducción de personal en las empresas con efectos negativos para las personas que pierden su trabajo y también para los «supervivientes», con un incremento de las bajas por enfermedad entre los trabajadores que se quedan en la empresa por intensificación del trabajo, de las exigencias emocionales, aumento de las jornadas o aumento de la inseguridad. También se ha observado un aumento de la mortalidad de los trabajadores que han conservado el trabajo causadas por dolencias cardiovascular en los meses y años posteriores a las reestructuraciones (EUROFOUND y otros, 2014; Vathera y otros, 2004).

\section{Destrucción de empleo}

La crisis iniciada en 2008 ha tenido un fuerte impacto en el empleo en Cataluña. De hecho, entre 2008 y 2013 se ha producido un descenso de la población ocupada del 17,1\% (un 20,1\% entre los hombres y un 8,5\% entre las mujeres) (DEMO, 2014).

En cuanto al trabajo asalariado, hay que remarcar que, en el mismo periodo, el grueso de la reducción de trabajadores afiliados a la Seguridad Social se ha producido en los tramos de edad correspondientes a los más jóvenes, tanto por la pérdida más intensa de los puestos de trabajo que ocupaban, como por la dificultad para incorporarse al mercado de trabajo. Así, la caída del número de afiliados menores de 25 años ha sido de más del $50 \%$, y superior al 40\% entre los trabajadores de entre 25 y 34 años (DEMO, 2014). La destrucción de empleo entre los más jóvenes se puede relacionar con el hecho de que la pérdida de empleo se ha producido en su mayoría no tanto por despido de trabajadores con contratos indefinidos, sino sobre todo por la no renovación de contratos temporales.

Por lo tanto, se puede concluir que la crisis ha golpeado con especial dureza a los más jóvenes, en unas edades en las que se empiezan (o así debería ser) a concretar los proyectos vitales, que sin el apoyo de una fuente de ingresos estable son en muchos casos inviables. No se puede menospreciar el impacto de este fenómeno en términos de bienestar y estabilidad psíquica entre la población más joven, ni tampoco el impacto que tendrá en el conjunto de la sociedad en el futuro.

La destrucción de empleo ha afectado fundamentalmente a las personas que tenían un contrato temporal. De acuerdo con los datos publicados por el Idescat, un 19,1\% de los hombres con contrato indefinido han perdido el puesto de trabajo, por solo un 4,5\% de mujeres. En cambio, la pérdida del puesto de trabajo entre los trabajadores temporales ha afectado a un $25 \%$ del total, tanto entre hombres como entre mujeres (DEMO, 2014).

\section{Más precariedad y más inseguridad relacionadas con el trabajo}

En cuanto a la calidad del empleo, hay varios indicadores que muestran que aquellas personas que han conservado el empleo trabajan hoy en peores condiciones que antes de la crisis.

En relación con el tiempo de trabajo, por ejemplo, la comparación entre la primera y la segunda Encuesta de condiciones de trabajo de Cataluña muestra que entre 2005 y 2010 (la tercera edición de la Encuesta catalana está planificada para 2016) se ha producido un incremento de más del 50\% en el número de personas que aseguran trabajar más de 48 horas semanales. También ha aumentado, aunque en menor medida, el número de personas que trabajan menos de 35 horas a la semana, que se puede considerar que no hacen una jornada completa. Hay que tener presente que en la mayoría de los casos el trabajo a tiempo parcial no es deseado, sino que los trabajadores lo aceptan porque no encuentran una alternativa a tiempo completo (DEMO, 2012).

En cuanto a las condiciones de seguridad y salud laboral, entre 2005 y 2010 se produce un aumento de las personas expuestas a todos los factores de riesgo ergonómicos identificados. Destaca en particular que se ha triplicado el porcentaje de trabajadores que indica que debe estirar los brazos para llegar a herramientas, elementos u objetos de trabajo (pasando del 5,4\% en el año 2005 al 15,6\% en 2010), y que se ha duplicado la proporción de 
trabajadores que deben mantener posturas forzadas con cualquier parte del cuerpo (pasando del 9,2\% en 2005 al 18,2\% en 2010). También es significativo el aumento de cerca del $50 \%$ en el porcentaje de trabajadores que tienen que trabajar con los brazos por encima de los hombros, que eran un 9,7\% en 2005 y pasaron a ser el 14,6\% en 2010 (DEMO, 2012).

En cuanto a los factores de riesgo psicosocial, de 2005 a 2010 se observa entre las personas asalariadas con contrato un aumento de las exigencias psicológicas del trabajo, tanto por la intensificación del trabajo como por un empeoramiento de todos los indicadores de control sobre el trabajo (Utzet y otros, 2014). Hay que tener presente que la combinación de elevadas exigencias psicológicas y un bajo nivel de control de los trabajadores sobre el propio trabajo produce lo que se denomina «alta tensión» y el cambio del patrón de las exposiciones psicosociales podría suponer un aumento de la población expuesta a condiciones psicosociales relacionadas con las enfermedades cardiovasculares o los trastornos de salud mental (Utzet y otros, 2015).

\section{Gráfico 1. Prevalencias de exposición al riesgo psicosocial de la población asalariada con contrato según sexo. Cataluña 2005-2010}

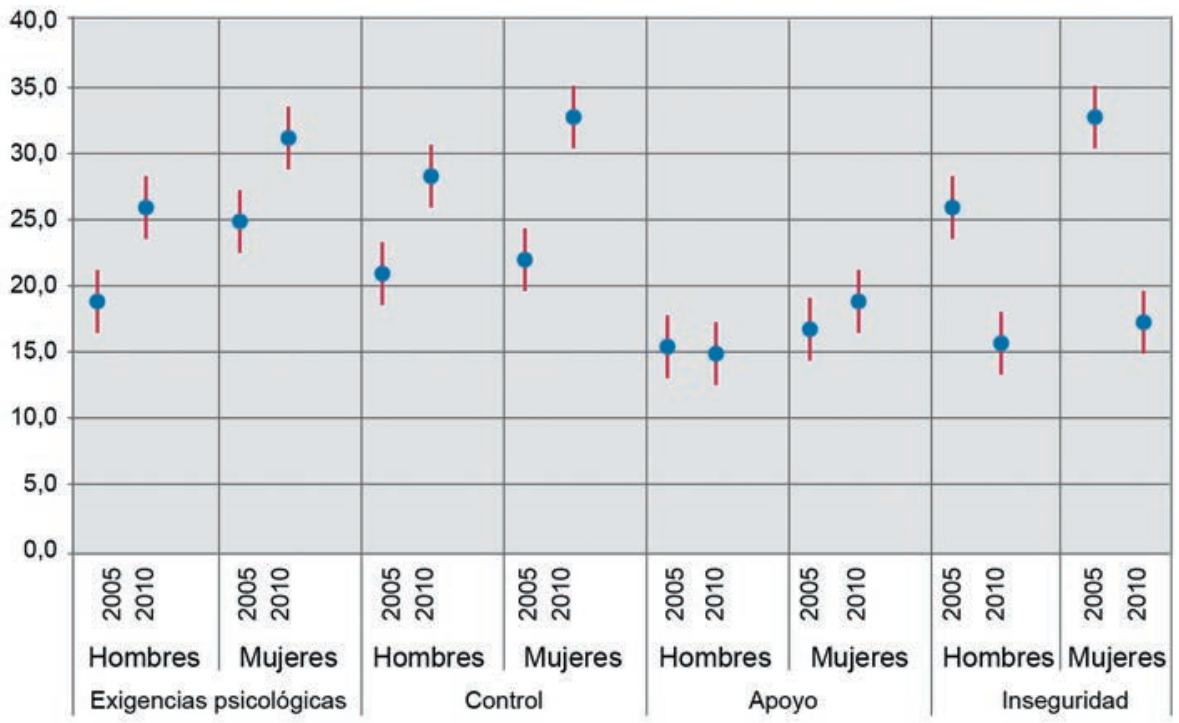

Fuente: Il Encuesta catalana de condiciones de trabajo (DEMO, 2012).

En el mismo periodo, como es de esperar, se observa un empeoramiento de la inseguridad sobre el empleo, pero, paradójicamente, no sobre las condiciones de trabajo (jornada, horarios, traslados de centro de trabajo, etc.) (DEMO, 2013). Este fenómeno se puede explicar porque la misma inquietud por perder el trabajo puede convertir en secundario el empeoramiento de condiciones salariales, de horario... u otros derechos, siempre que se conserve el trabajo, que es el bien esencial en momentos en los que el empleo está en peligro. Este aumento de la exposición a alta inseguridad ha sido ampliamente relacionado con problemas de salud (Lászlo y otros, 2010; Ferrie y otros, 2005, 2008).

Vinculado a esto hay que reseñar que en 2014 solo el 25\% de las empresas de más de diez trabajadores contaban con delegados de prevención en Cataluña, aunque se pueden convocar elecciones a representantes de los trabajadores incluso a iniciativa de los sindicatos. En las empresas de seis a diez trabajadores, donde la plantilla debe decidir por mayoría si quiere celebrar o no elecciones para elegir a sus representantes, solo un 4,4\% tenían delegado de prevención (que también es delegado de personal) (DEMO, 2015).

Este bajo nivel de representación de los trabajadores en las empresas, que se produce en particular en aquellas que tienen hasta 250 trabajadores (por encima de esta cifra alrededor del 90\% de las empresas tienen representantes de los trabajadores), no tiene una relación directa con la crisis, pero sí que sería un factor de debilitamiento ante el deterioro de las condiciones de trabajo. 
Es lógico pensar que si la inseguridad en las condiciones de trabajo ha bajado, es decir, que los cambios unilaterales realizados por la empresa han preocupado menos durante la crisis, al quedar todo supeditado a la conservación del trabajo, aún con más razón los trabajadores no se sentirán suficientemente confiados como para reclamar mejoras de las condiciones de trabajo en relación con su seguridad y salud.

El hecho de que en la inmensa mayoría de las empresas de Cataluña no exista representación de los trabajadores, gozando de la protección que la normativa reconoce a los delegados de personal y miembros de comités de empresa, significa que no hay en los centros de trabajo figuras que puedan plantear estas demandas sin sentir que corren un riesgo en relación con la conservación de su empleo. Es difícil valorar cuantitativa o cualitativamente la incidencia de esta falta de representación de los trabajadores especializada en prevención, pero si hay consenso en que la figura del delegado de prevención contribuye a la mejora de la seguridad y salud en la empresa, convendremos que su ausencia hace que, como mínimo, no se produzcan las mejoras que los delegados de prevención impulsan (Menéndez y otros, 2009; Reilly y otros, 1995; Coutrot, 2009).

\section{Crisis y siniestralidad laboral}

Una mirada excesivamente simplista sobre la relación entre crisis y condiciones de trabajo se limitaría a mirar si durante el periodo de graves dificultades económicas se ha producido o no un aumento en el número de accidentes de trabajo y enfermedades profesionales, o mejor dicho, en su tasa de incidencia (número de accidentes por cada 100.000 trabajadores afiliados a la Seguridad Social). Para ello bastaría con analizar la evolución de los datos de siniestralidad laboral desde 2007 hasta la actualidad.

Gráfico 2. Índice de incidencia de accidentes en jornada de trabajo con baja. Cataluña 2007-2014

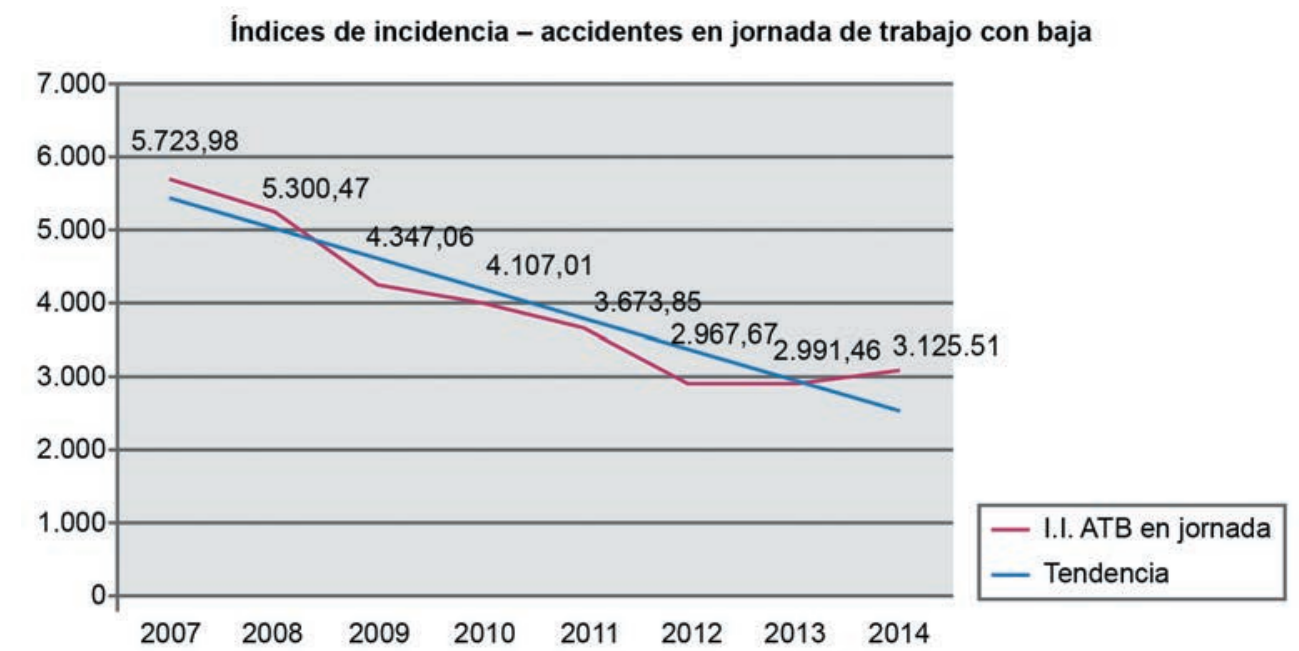

Fuente: Departamento de Empresa y Empleo. 


\section{Gráfico 3. Índice de incidencia de accidentes mortales en jornada de trabajo. Cataluña 2007-2014}

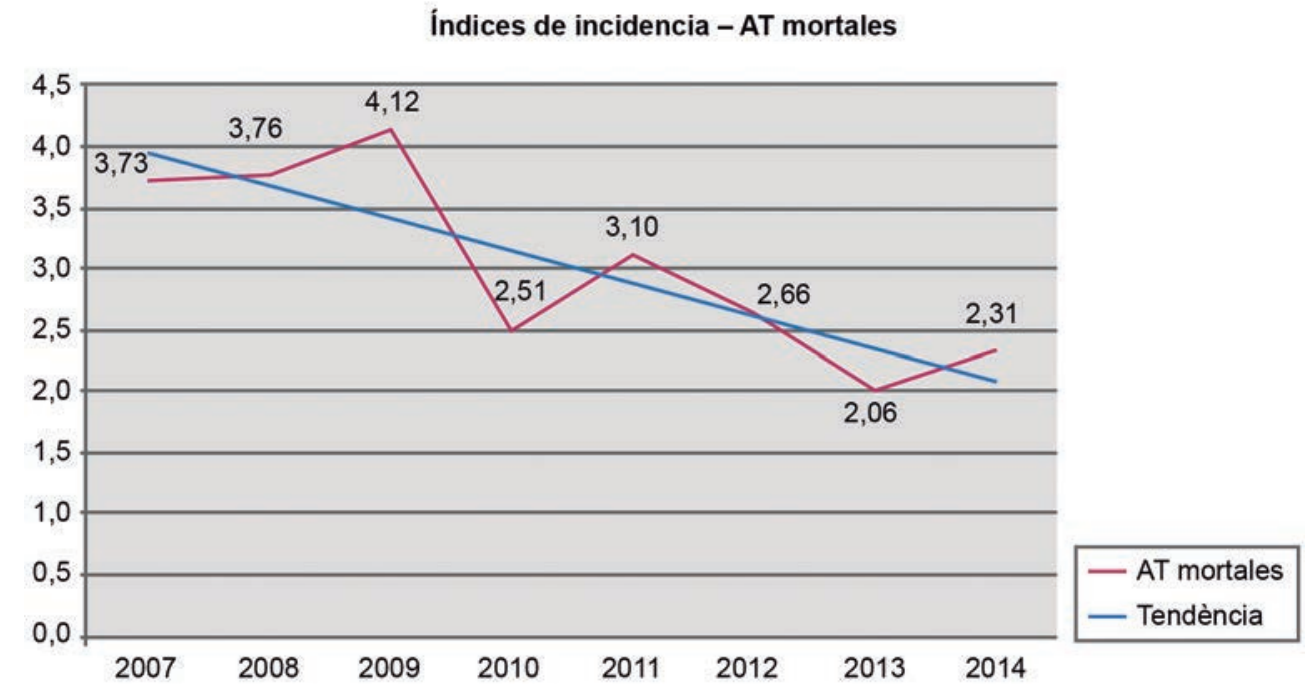

Fuente: Departamento de Empresa y Empleo.

Entendemos, sin embargo, que esta operación nos puede llevar a conclusiones equívocas, por varias razones que hay que explicitar.

En primer lugar, hay que ser conscientes de las limitaciones de los registros públicos en materia de accidentes de trabajo y enfermedades laborales (consideradas profesionales o no) para reflejar los daños a la salud derivados del trabajo. Los conceptos de accidente de trabajo y enfermedad profesional provienen, más que del mundo de la seguridad y la salud en el trabajo, del ámbito de la Seguridad Social. Ambos conceptos tienen la función primordial de delimitar en qué casos los trabajadores pueden acceder a las prestaciones reforzadas (mayor importe económico, no exigencia de periodos mínimos de cotización previa para tener derecho a la prestación, etc.) que se ofrecen cuando la causa del accidente o enfermedad que sufra un trabajador es el trabajo.

Así, no todos los accidentes de trabajo registrados tienen su causa directa en unas condiciones de trabajo inadecuadas (el ejemplo más claro es el de los accidentes in itinere). En cambio, los actos intencionales, como el suicidio, y más si tienen lugar fuera del lugar de trabajo y fuera de su jornada, se considera que no son accidentes de trabajo. Esto, por ejemplo, impide registrar como accidentes de trabajo suicidios que pueden tener una causa laboral.

Para aquellas dolencias o daños a la salud que se produzcan fuera del lugar de trabajo y que no estén incluidas en la lista de enfermedades profesionales es necesario probar que tienen un origen en el trabajo que desarrolla la persona. Este hecho, al que se añaden las dificultades y desinformación de los trabajadores enfermos y los médicos del sistema asistencial sobre la necesidad y conveniencia de identificar y comunicar el posible origen profesional de una patología que se manifiesta fuera del trabajo, hace que muchos daños a la salud derivados del trabajo pasen inadvertidos y no queden contabilizados.

Aparte de eso, hay que tener presente que la crisis ha provocado una profunda modificación del mercado de trabajo, al haber afectado con mucha mayor dureza a sectores como la construcción, donde los índices de siniestralidad duplican o triplican a los del resto de los sectores. Así, el hecho de que en proporción haya muchos menos albañiles, encofradores, yeseros, etc., entre la población ocupada se traduce en una reducción del número de accidentes de trabajo importante e, incluso, en una reducción de los índices de incidencia globales (no los de sector económico). 
Por otra parte, tenemos un gran desconocimiento de los daños a la salud relacionados con las exposiciones laborales, que, a diferencia de los accidentes de trabajo, tienen un tiempo de latencia largo entre esta exposición y el momento del diagnóstico del problema de salud. Este aspecto, ya importante en momentos de bonanza económica, lo es mucho más en relación con las situaciones de crisis y los daños que se originan por exposiciones en el periodo actual pero que se manifestarán en el futuro. Estos daños son fundamentalmente patologías musculoesqueléticas, cardiovasculares y de salud mental.

Este fenómeno puede provocar un cierto efecto «espejismo", es decir, que los datos muestren una reducción de los accidentes de trabajo y enfermedades profesionales, pero que en la práctica se haya producido un importante deterioro de las condiciones de trabajo de los trabajadores ocupados. Este deterioro no necesariamente se traduce, al menos a corto plazo, en un incremento de accidentes de trabajo o enfermedades profesionales «contabilizables", pero no por ello es menos real.

Para decirlo de forma caricaturesca, pero comprensible, quizá en estos años hemos perdido muchos trabajadores de la construcción, que sufrían accidentes con mucha frecuencia e inequívocamente de trabajo porque se producían en la obra, pero tenemos trabajadores del resto de los sectores sometidos a una fuerte sobrecarga de trabajo, inseguridad laboral, etc. Estos factores de riesgo psicosocial no producen consecuencias inmediatas, y cuando se concretan en un daño a la salud es más probable que su origen laboral pase inadvertido que cuando un albañil, por ejemplo, se hace un corte o cae de un andamio.

\section{Conclusiones}

Los efectos de la crisis en las condiciones de trabajo y en muchos otros aspectos relacionados con el trabajo son indudables y muy intensos; tenemos mucha información que lo acredita. En los siete años que llevamos de deterioro económico se ha producido un incremento notable de las exigencias a los trabajadores, una disminución de las contrapartidas que estos reciben y un cierto destierro de la prevención de riesgos laborales en términos de atención empresarial y de inversión. No vale la pena analizar las posibles justificaciones -siempre habrá quien aduzca que en situaciones extremas la prioridad es la supervivencia-, pero lo cierto es que, como ocurre con nuestro cuerpo, unos malos hábitos de salud sostenidos en el tiempo al final acaban, indefectiblemente, por pasar factura.

Es muy posible que este más que probable destierro de la prevención de las prioridades empresariales aún no se haya materializado en unos resultados negativos en forma de accidentes de trabajo o enfermedades profesionales porque la evolución en este ámbito es lenta. Una máquina de la que no se hace un mantenimiento adecuado, para ahorrar costes, no se estropea o provoca un accidente al día siguiente. Seguramente podrá seguir funcionando con aparente normalidad un tiempo, más o menos prolongado, pero al final esta desatención tendrá consecuencias.

Por el contrario, no sería descabellado pensar que el hecho de que la siniestralidad, especialmente por accidentes de trabajo, haya mantenido durante la crisis la evolución positiva que presentaba los años previos de bonanza se debe a que las inversiones y los esfuerzos hechos en prevención, tanto en equipamiento como en formación de los trabajadores y otros aspectos organizativos, han podido mantener su eficacia durante un tiempo. Esto, junto con las consideraciones expuestas más arriba, puede explicar que el empeoramiento de condiciones de trabajo no haya comportado un mayor número de accidentes en un primer momento.

Son precisamente todas estas razones las que nos llevan a subrayar con mucho énfasis la necesidad de que las empresas, ahora que parece que estamos entrando en un punto de inflexión en cuanto a la evolución de la economía y se divisa una cierta recuperación, den prioridad a la inversión en prevención. Es importante recuperar cuanto antes el terreno perdido en esta materia durante estos años de crisis.

Todavía estamos a tiempo de evitar que la menor preocupación por la seguridad y la salud de los trabajadores nos pase factura en forma de accidentes de trabajo y enfermedades laborales, pero sería un inmenso error pensar que aquella máquina deteriorada aún puede aguantar un poco más, o que aquella iniciativa de formación puede esperar hasta el año que viene, o el otro. No, ya estamos en tiempo de prórroga, y si no hacemos los deberes ahora, como se dice en el argot futbolístico, nos lo jugaremos todo «en la lotería de los penaltis». Sí, lotería, ese antónimo de prevención. 


\section{Bibliografía}

COUTROT, T. (2009). "Le rôle des comités d'hygiène, de sécurité et des conditions de travail en France: Une analyse empirique». Travail Et Emploi. Núm. 117, pág. 25-38.

DEMO (2012). Segona enquesta catalana de condicions de treball. Barcelona: Dirección General de Relaciones Laborales y Calidad en el Trabajo. Departamento de Empresa y Empleo. Generalitat de Catalunya [publicación en línea]. [Fecha de consulta: 10 de abril de 2015]. http://empresaiocupacio.gencat.cat/web/.content/03_-_centre_de_documentacio/documents/01_-_publicacions/06_-_seguretat_i_salut_laboral/ii_ecct/ arxius/enquesta_treballadors.pdf

DEMO (2013). Evolució de l'exposició a riscos psicosocials de la població assalariada amb contracte a Catalunya entre 2006 i 2010. Barcelona: Dirección General de Relaciones Laborales y Calidad en el Trabajo. Departamento de Empresa y Empleo. Generalitat de Catalunya [publicación en línea]. [Fecha de consulta: 10 de abril de 2015]. http://empresaiocupacio.gencat.cat/web/.content/03_-_centre_de_documentacio/documents/01_-_publicacions/06_-_seguretat_i_salut_laboral/ii_ecct/arxius/evolucio_exposicio_riscos_psicosocials_2006_2010.pdf

DEMO (2014). Indicadors de seguretat i salut en el treball. Catalunya 2008-2013. Barcelona: Área de Investigación. Subdirección General de Seguridad y Salud Laboral. Departamento de Empresa y Empleo. Generalitat de Catalunya [publicación en línea]. [Fecha de consulta: 10 de abril de 2015]. http://empresaiocupacio.gencat.cat/ web/.content/03_-_centre_de_documentacio/documents/01_-_publicacions/06_-_seguretat_i_salut_laboral/ arxius/Indicadors_SSL_CAT_2008_2013.pdf

DEMO (2015). Gestió de la prevenció de riscos laborals a les empreses de Catalunya 2014. Área de Investigación. Subdirección General de Seguridad y Salud Laboral. Departamento de Empresa y Empleo. Generalitat de Catalunya. Pendiente de publicación.

EUROFOUND; OSHA (2014). Psychosocial risks in Europe: Prevalence and strategies for prevention. Luxemburgo: Publications Office of the European Union.

FERRIE, J. E.; SHIPLEY, M. J.; NEWMAN, K.; STANSFELD, S. A.; MARMOT, M. (2005). «Self-reported job insecurity and health in the Whitehall II study: potential explanations of the relationship». Soc Sci Med. Vol. 60, núm. 7, pág. 1593-1602.

FERRIE, J. E.; WESTERLUND, H.; VIRTANEN, M.; VAHTERA, J.; KIVIMÄKI, M. (2008). «Flexible labor markets and employee health». Scand J Work Environ Health. Vol. 6 (supl.), pág. 98-110.

LÁSZLO, K. D.; PIKHART, H.; KOPP, M. S.; BOBAK, M.; PAJAK, A.; MALYUTINA, S,; SALAVECZ, G.; MARMOT, M. (2010). "Job insecurity and health: a study of 16 European countries». Soc Sci Med. Vol. 70, núm. 6, pág. 867-874.

MENÉNDEZ, M.; BENACH, J.; VOGEL, L. (2009). The impact of safety representatives on occupational health. A European perspective. Bruselas: ETUI [publicación en línea]. [Fecha de consulta: 10 de abril de 2015]. http://osha.europa.eu/en/news/eu-etui-report-the-impact-of-safety-representatives-on-occupational-health.a-european-perspective

REILLY, B.; PACl, P.; HOLL, P. (1995). «Unions, Safety Committees and Workplace Injuries». British Journal of Industrial Relations. Vol. 33, núm. 2, pág. 275-288.

RUGULIES, R.; AUST, B.; BURR, H.; BÜLTMANN, U. (2008). «Job insecurity, chances on the labour market and decline in self-rated health in a representative sample of the Danish workforce». J Epidemiol Community Health. Vol. 62, núm. 3, pág. 245-50.

UTZET, M.; MONCADA, S.; MOLINERO, E.; LLORENS, C.; MORENO, N.; NAVARRO, A. (2014). «The changing patterns of psychosocial exposures at work in the South of Europe: Spain as a labor market laboratory». Am J Ind Med. Vol. 57, pág. 1032-1042.

UTZET, M.; NAVARRO, A.; LLORENS, C.; MONCADA, S. (2015). «Intensification and isolation: changes in the psychosocial work environment in Spain between 2005 and 2010». Occup Med. Pendiente de publicación.

VATHERA, J.; KIVIMÄKI, M.; PENTTI, J.; LINNA, A.; VIRTANEN, M.; VIRTANEN, P.; FERRIE, J. E. (2004). «Organisational downsizing, sickness absence, and mortality: 10-town prospective cohort study». BMJ. Vol. 328, núm. 7439, pág. 555. 


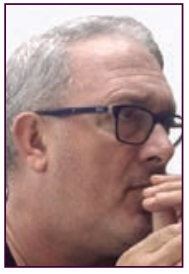

\section{Jaume de Montserrat i Nonó}

jdemontserrat@gencat.cat

Instituto de Seguridad y Salud Laboral. Departamento de Empresa y Empleo de la Generalitat de Cataluña

Licenciado en Medicina y Cirugía por la Universidad Autónoma de Barcelona; especialista en Medicina del Trabajo, diplomado en Medicina de Empresa y técnico superior de Prevención de Riesgos Laborales en las especialidades de Ergonomía y Psicosociología aplicada, Higiene Industrial y Seguridad en el Trabajo.

En el año 1987 entró a trabajar en el Centro de Seguridad y Salud Laboral de Girona del Departamento de Trabajo de la Generalitat (actualmente Departamento de Empresa y Empleo), del cual fue director desde 1997 hasta junio de 2008, momento en el que fue nombrado subdirector general de Seguridad y Salud Laboral del Departamento de Empresa y Empleo (DEMO, por sus siglas en catalán) de la Generalitat de Cataluña, cargo que ocupa actualmente.

Paralelamente a su trabajo en el DEMO, está vinculado a diversas sociedades científicas, como la Sociedad Catalana de Seguridad y Medicina del Trabajo, que presidió entre 1997 y 2001. Tiene una presencia activa en diversas publicaciones de salud laboral y es ponente habitual en congresos de salud laboral. Desarrolla amplias tareas de docencia relacionadas con la salud laboral y es profesor asociado de la Universidad de Girona, en el Área de Salud Laboral de la Escuela Universitaria de Enfermería.

\section{Fmilia Molinero Ruiz emilia.molinero@gencat.cat Instituto de Seguridad y Salud Laboral. Departamento de Empresa y Empleo de la Generalitat de Cataluña}

Licenciada en Medicina y Cirugía por la Universidad Autónoma de Barcelona; especialista en Medicina del Trabajo, diplomada en Medicina de Empresa y técnico superior de Prevención de Riesgos Laborales en las especialidades de Ergonomía y Psicosociología aplicada (máster por la Universidad Pompeu Fabra). Máster en Metodología de la investigación en ciencias de la salud (Universidad Autónoma de Barcelona). Dedicada profesionalmente a la salud laboral desde 1985: médico del trabajo en una administración local hasta 1999, posteriormente desarrolló su actividad laboral en el Servicio de Salud Laboral de la Agencia de Salud Pública de Barcelona y, desde 2009, en la Subdirección General de Seguridad y Salud Laboral como responsable del Área de Investigación, con una presencia activa en diversas publicaciones de salud laboral.

\section{Julià Nájera Chico}

jnajera@gencat.cat

Instituto de Seguridad y Salud Laboral. Departamento de Empresa y Empleo de la Generalitat de Cataluña

Licenciado en Medicina y Cirugía por la Universidad de Barcelona; máster en Informática sanitaria y técnico superior de Prevención de Riesgos Laborales en la especialidad de Ergonomía y Psicosociología aplicada por el IdEC-ISP.

En el año 1992 se incorporó al Centro de Seguridad y Salud Laboral de Lleida del Departamento de Trabajo (actualmente Departamento de Empresa y Empleo), donde ejerció como médico en la sección de Medicina.

En el año 2005 se incorporó al equipo de la Subdirección General de Seguridad y Salud Laboral del Departamento de Trabajo, y en el año 2008 se hizo cargo del Área de Información y Registros de Seguridad y Salud Laboral de esta Subdirección, tarea que sigue desarrollando en la actualidad. 


\section{Andrés Ros Pueyo}

andres.ros@gencat.cat

Instituto de Seguridad y Salud Laboral. Departamento de Empresa y Empleo de la Generalitat de Cataluña

Licenciado en Periodismo por la Universidad Pompeu Fabra de Barcelona y en Historia por la Universidad de Barcelona, trabajó en diversas agencias de noticias y gabinetes de prensa. En el año 2006 se incorporó al Gabinete de Prensa del Departamento de Trabajo e Industria (actualmente Departamento de Empresa y Empleo). Tras un breve periodo integrado en la Dirección General de Relaciones Laborales, en el año 2010 se incorporó a la Subdirección General de Seguridad Laboral, donde desarrolla tareas de edición y gestión de publicaciones sobre seguridad y salud laboral, así como de elaboración de contenidos para la web y redes sociales.

\section{Jiri Turdy i Moix jiri.turdy@gencat.cat Instituto de Seguridad y Salud Laboral. Departamento de Empresa y Ocupación de la Generalitat de Cataluña}

Ingeniero Técnico Industrial por la Universidad Politécnica de Cataluña, ingeniero de Organización Industrial por la Universidad Politécnica de Cataluña y técnico superior de Prevención de Riesgos Laborales en las especialidades de Seguridad en el Trabajo, Higiene Industrial y Ergonomía y Psicosociología aplicada.

En el año 1992 entró a trabajar en el Centro de Seguridad y Salud Laboral de Barcelona del Departamento de Trabajo de la Generalitat de Cataluña (actualmente Departamento de Empresa y Empleo). En el año 2004 fue adscrito a la Subdirección General de Seguridad y Salud Laboral del Departamento de Empresa y Empleo. Desde el año 2008 es el jefe del Área Técnica.

Los textos publicados en esta revista están -si no se indica lo contrario- bajo una licencia Reconocimiento-Sin obras derivadas 3.0 España de Creative Commons. Puede copiarlos, distribuirlos y comunicarlos públicamente siempre que cite su autor y la revista y la institución que los publica (autoría, nombre de la revista, institución editora); no haga con ellos obras derivadas. La licencia completa se puede consultar en http://creativecommons.org/licenses/by-nd/3.0/es/deed.es.

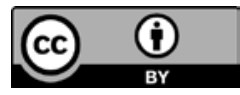

